\title{
ReSCUE: Relatively Stable Clustering for Unbiased Environments in VANETs
}

\author{
Muhammed Nur Avcil \\ Department of Computer Engineering \\ Marmara University \\ Istanbul, Turkey 34722 \\ Email: muhammed.avcil@marmara.edu.tr
}

\author{
Mujdat Soyturk \\ Department of Computer Engineering \\ Marmara University \\ Istanbul, Turkey 34722 \\ Email: mujdat.soyturk@marmara.edu.tr
}

\begin{abstract}
Clustering algorithms improve network manageability through several topology partitioning techniques. In some particular cases, such as vehicular ad hoc network (VANETs) communications, significant performance improvements can be introduced via clustered networking solutions whereas merging clusters for the sake of scalability may lead to degraded network stability. In this paper, we explore the impact of merging clusters, and furthermore based on these results, we propose a new clustering technique, namely Relatively Stable Clustering for Unbiased Environments (ReSCUE). The objective of ReSCUE is primarily guaranteeing cluster stability in an unbiased manner. ReSCUE keeps track of the spatio-temporal changes in VANET node characteristics, and uses these characteristics along with local information to prevent biased clustering which is based on common and general node characteristics. We evaluate the performance of ReSCUE through simulations and show that ReSCUE can form relatively more stable clusters while reducing the frequency of cluster merges, as well as that of the node status changes.
\end{abstract}

Keywords-Clustering, stability, discrimination, merge, VANET

\section{INTRODUCTION}

Vehicular Ad Hoc Networks (VANETs) have unique characteristics such as having highly mobile nodes and frequent topology changes which cause intermittent connectivity and poor communication between nodes [1]. Frequent density and speed variations in time and space worsen the situation. Clustering is used to mitigate this problem by partitioning the networks into groups of nodes composed of similar characteristics.

Forming stable clusters and avoiding frequent cluster head $(\mathrm{CH})$ change are the two major concerns in clustering. On the other hand, the studies in the literature [2] [3] [4] [5] [6], generally enforce clusters to merge to form a single cluster when the clusters moving in the same direction meet with each other. However, this general approach disregards an important fact in clustering. Formerly in the network, clusters were formed considering the common characteristics of the vehicles. Vehicles in separate clusters usually show different characteristics e.g. speed. Merging the clusters that catches up the slow one disregards this principal feature. After a while due to speed differences, the faster cluster will overtake the slower one, and the nodes will split off to re-form new clusters. In these streaking consecutive events, $\mathrm{CHs}$ are reelected and the nodes reselect and reregister to their new CHs. Moreover, these consecutive events introduces additional overhead.

In this paper, we propose a new clustering approach which aims to form more stable clusters considering multiple criteria and parameters of the nodes. In addition to the stable clustering, the proposed approach also aims to prevent clusters from merging if the conditions are not suitable to merge or merging causes disadvantages afterwards. Approaches in the literature generally tend to form clusters based on common characteristics and similarities of the nodes. In addition to the similarities, the proposed approach also considers the differences in the characteristics of the vehicles and the clusters. It is a key to form more stable clusters in highly mobile environment.

Another important feature of the proposed approach is the use of local information to avoid biased clustering due to the use of globally known general information. Clustering the vehicles based on their well-known and general characteristics induces biased cluster formation which does not reflect the current conditions and characteristics of the nodes. Vehicle characteristics e.g. speed show changes in time and space. For example, a speedy automobile may sometimes show similar characteristics with a truck by slowing down on climbing hills, etc. Rather than considering general and out-of-date characteristics of the vehicles, recent and up-to-date characteristics have to be considered to reflect the changing conditions at nodes and in the network. The proposed approach considers the locally collected recent information to discriminate the characteristics of the vehicles to provide unbiased clustering in the current environmental conditions.

In addition to forming more stable clusters, other goals are minimizing the number of cluster heads, minimizing the cluster head change, maximizing the duration of cluster head and minimizing the overhead in forming the clusters. To achieve these goals, the proposed approach uses multiple parameters including direction, position, density, speed and standard deviation of the speed of the vehicles. Each vehicle share these information with its neighbors. On these parameters, speed and standard deviation of the speed are used to distinguish vehicles based on their speed characteristics. Other parameters are used in $\mathrm{CH}$ election. Cluster formation and $\mathrm{CH}$ election is achieved distributedly without incurring addition delay. Formed clusters with vehicles based on similar characteristics are more stable and live longer period of time. 
The rest of study is organized as follows. Section 2 describes the related works on clustering algorithms. The proposed clustering method is described in Section 3. In Section 4, the simulation environment and performance results are given in addition to the comparison with similar approaches. Finally, Section 5 concludes the paper.

\section{RELATED WORK}

In the literature, two well-known clustering algorithms for mobile ad hoc networks are the Lowest-ID [3] and the HighestDegree [2]. In Lowest-ID, each node has a unique ID which is used in Cluster Head $(\mathrm{CH})$ selection. The node having lowest ID in its neighborhood is elected as the $\mathrm{CH}$. There are two enhanced versions of Lowest-ID, namely the Least Cluster Head Change (LCC) [7] and the Random Competition based Clustering (RCC) [8]. These two enhanced versions introduce the $\mathrm{CH}$ role give up approach when two $\mathrm{CHs}$ move within their transmission range. Only one of the $\mathrm{CHs}$ continue to serve a $\mathrm{CH}$. In the Highest-Degree [2] algorithm, the $\mathrm{CH}$ is elected considering the number of connections of the nodes. The node which has the maximum number of neighbors in its transmission range is elected as the $\mathrm{CH}$. Lowest-ID and Highest-Degree are two preliminary approaches proposed for MANET. Though simple to implement, they suffer and perform poor in high mobile environments where the topology changes rapidly.

MOBIC [4], N-Hops [6] and ALM [5] are the clustering methods which consider the relative mobility. Although these approaches are very similar to each other, the relative mobility metric varies. MOBIC uses the received signal strength (RSS), $\mathrm{N}$-Hops uses the packet delay and ALM uses the distance as the relative mobility metric. The relative mobility is measured considering two successive messages from the same neighbor. $\mathrm{CH}$ selection is based on calculation of aggregate local mobility which is later compared to the relative mobility value. The node with the lowest aggregate value is elected as the $\mathrm{CH}$. NHops is a multi-hop clustering method which as well as ALM are proposed for VANETs (Vehicular Ad Hoc Networks).

Another recent clustering method designed for VANETs is the Threshold Based (TB) [9] clustering method. Clusters are formed and $\mathrm{CHs}$ are elected considering the mobility of the nodes and the relative lower and higher speed neighbor node lists. Cluster formation and $\mathrm{CH}$ election are initiated once at the beginning by one of the nodes. After the first cluster formation and $\mathrm{CH}$ selection, $\mathrm{CH}$ election is not initiated anymore and clusters are not formed periodically afterwards. Nodes may join and leave the cluster but the $\mathrm{CH}$ doesn't change. $\mathrm{CH}$ election method is evoked only when multiple $\mathrm{CHs}$ meet with each other to join the clusters and to reduce the number of $\mathrm{CH}$ to one. Compared to the other approaches in the literature, TB considers more parameters to form more stable clusters in the cluster formation phase. However, it doesn't reflect the changing conditions afterwards. CHs don't change even nodes join and leave the clusters. Stability of the clusters and best suitable node feature of the $\mathrm{CHs}$ are not met anymore.

In most of the proposed approaches in the literature, cluster head selection and cluster formation are initiated on merging clusters into one cluster when two or more clusters meet with each other. However, more likely the merged cluster will split
TABLE I. NOTATION

\begin{tabular}{cl}
\hline Notation & Description \\
\hline$C_{i}$ & Coherence value for node $n_{i}$ \\
$S C N$ & Similar characteristics node \\
$S C N_{i}$ & Similar characteristic node list of node $n_{i}$ \\
$N_{i}^{n e i g h}$ & Number of the neighbor nodes of node $n_{i}$ \\
$N_{i}^{S C N}$ & Number of the neighbor nodes showing similar char- \\
$\Delta t$ & acteristics with node $n_{i}$ \\
$t_{i}^{w a i t}$ & Time period for sampling the speed \\
$V_{i}(t)$ & Backoff time for node $n_{i}$ to declare itself as CH \\
$V_{i}$ & Mean value of the speeds of node $n_{i}$ during $\Delta t$ \\
$s_{i}^{v}$ & Standard deviation of the speeds of node $n_{i}$ during \\
$P_{i}(t)$ & $\Delta t$ \\
$z_{i}^{v}$ & The position of node $n_{i}$ at time $t$ \\
$z_{i}^{p}$ & Normalized speed value of node $n_{i}$ \\
$M s g_{i}^{C H}$ & Normalized position value of node $n_{i}$ \\
$C H$ & Cluster Head declaration message of node $n_{i}$ \\
$C H$ & Cluster Head \\
$C M$ & Cluster Head node of node $n_{i}$ \\
Non-Clustered & Cluster Member \\
\hline & Ordinary node which is not a member of any cluster \\
\hline
\end{tabular}

off again due to the characteristic differences of the nodes e.g. speed. Fast moving nodes will reinitiate the cluster formation and $\mathrm{CH}$ selection when they are overtaking the slow moving vehicles. These conditions introduce messaging overhead and unnecessary status changes at nodes. One of our aim in this research is avoiding unnecessary cluster joins and thus status changes.

\section{The Proposed ApProACH}

In highways, vehicles usually tend to move in groups. The prominent example is the trucks at highways which usually drive in the right most lane and with a slower speed compared to other vehicles. Cars move in faster speeds and some cars move much faster compared to most of the vehicles. The proposed approach aims to differentiate groups based on this main characteristic, namely the speed and its variation in time. In each differentiated group, speeds of the vehicles can be modeled with normal distribution with mean $\mu_{\text {class }_{k}}^{v}$ and standard deviation $\sigma_{\text {class }_{k}}^{v}$ [10] [11], if there are $k$ number of vehicle groups. However, actual speed parameters $\left(\mu_{\text {class }_{k}}^{v}\right.$ and $\sigma_{\text {class }_{k}}^{v}$ ) of the vehicle groups are unknown and cannot be modeled and derived instantly in a highly changing environment. Moreover, the number of groups and their characteristics may change in time and space. To select most coherent group, a node can utilize the local information obtained from its neighborhood. Information belonging to the the neighborhood can be used to determine the speed and other characteristics of the vehicle groups in nearby. Based on the sample information (local neighborhood information), actual parameters of the groups can be estimated and a node can determine the most appropriate group of nodes that it shows similar characteristics. Moreover, the use of local sampled information is much more convenient rather than using actual (global) predetermined values because a vehicle individually may show different characteristics depending on time and space for any reasons. The use of local information, the similarities and differences 
of the nodes in the formation of the clusters are the major differences from the other approaches in the literature.

If each vehicle records its recent speed values $V(t)$ for the time interval $\Delta t$, these speed values can be modeled with normal distribution or $t$-distribution with a mean value $\overline{V_{i}}$ and standard deviation $s_{i}^{v}$ as given in (1) and (2) respectively. These information is used in discrimination of vehicles and groups in terms of speed.

$$
\begin{gathered}
\overline{V_{i}}=\frac{1}{\Delta t} \sum_{t=1}^{\Delta t} V_{i}(t) \\
s_{i}^{v}=\sqrt{\frac{1}{\Delta t-1} \sum_{t=1}^{\Delta t}\left(V_{i}(t)-\overline{V_{i}}\right)^{2}}
\end{gathered}
$$

In the proposed approach, each vehicle updates its speed records continuously for the time period $\Delta t$. In beaconing periods, each vehicle calculates the standard deviation of its speed vector and insert this information into the beacon message in addition to its current speed, current position and direction. Upon reception of beacons, each vehicle updates the information about its neighbors. On clustering period, each vehicle performs (1) discrimination and distinguish of vehicles with similar characteristics based on speed to form a cluster, (2) calculation of a coherence value to contend to be $\mathrm{CH}$. These two methods are described below.

\section{A. Distinguishing Similar Characteristic Vehicles for Cluster Formation}

The main aim in this method is forming clusters with the nodes in similar characteristics $\left(S C N_{i}\right)$ in terms of speed. Since each node knows its mean velocity and its current speed, it can determine the $S C N_{i}$ by using the standard deviation of the speeds of neighbor nodes. Among the nodes in its neighbor list, the maximum standard deviation of speed, $s_{i, \max }^{v}$, is determined (3).

$$
s_{i, \max }^{v}=\max \left\{s_{i}^{v}, s_{j}^{v} \mid j=1,2 \ldots N_{i}^{\text {neigh }}\right\}
$$

where $N_{i}^{\text {neigh }}$ is number of neighbors within range of node $n_{i}$.

Each node determines the upper and lower bounds on the speed of similar vehicles using (4) and (5):

Upper Bound on the speed (for similar vehicles) of node $n_{i}$;

$$
U B_{i}^{v}=V_{i}+3 s_{i, \max }^{v}
$$

Lower Bound on the speed (for similar vehicles) of node $n_{i}$;

$$
L B_{i}^{v}=V_{i}-3 s_{i, \max }^{v}
$$

Each node determines its $S C N_{i}$ among the neighbor nodes $N_{i}^{\text {neigh }}$ which has speed values between these boundary values, $U B_{i}^{v}$ and $L B_{i}^{v}$. The number of nodes in $S C N_{i}$ of $n_{i}$ gives the $N_{i}^{S C N}$. In other words, $N_{i}^{S C N}$ is the number of nodes which have similar characteristic with node $n_{i}$ in terms of speed. Node $n_{i}$ will start forming cluster only with its neighbor nodes in its $S C N$ list.

\section{B. Calculation of Coherence Value to Contend to be $\mathrm{CH}$}

Next step is the election of $\mathrm{CH}$ in a distributed manner. In the re-clustering phase, each node calculates a coherence value to contend to be a $\mathrm{CH}$. Coherence value is used as a delay parameter in the declaration to be $\mathrm{CH}$. The node with highest coherence value announces itself as a $\mathrm{CH}$ earlier than others by suppressing them. On the other hand, $\mathrm{CH}$ selection plays the major role to form more stable clusters. Therefore, in the proposed approach multiple criteria is considered in the selection of $\mathrm{CH}$ and the calculation of the coherence value. The coherence value $C_{i}$, is derived considering the speed, position and local density of the node (6);

$$
C_{i}=\frac{N_{i}^{S C N}}{\mathrm{e}^{w_{i}}}
$$

where $w_{i}$ is a measure of the speed and position values of node $n_{i}$. In order to derive an unbiased coherence value (to be fair in the suppression and for accurate results), the speed and position values of node $n_{i}$ are normalized as defined in (7) and (8).

$$
\begin{aligned}
& \bar{x}_{i}^{v}=\frac{1}{N_{i}^{S C N}} \sum_{j=1}^{N_{i}^{S C N}} V_{j} \\
& \bar{x}_{i}^{p}=\frac{1}{N_{i}^{S C N}} \sum_{j=1}^{N_{i}^{S C N}} P_{j}
\end{aligned}
$$

where $\bar{x}_{i}^{v}$ and $\bar{x}_{i}^{p}$ are the mean values of the speed and the position of node $n_{i}$ for its $S C N_{i}$ respectively.

$$
\begin{aligned}
s_{i}^{v, S C N_{i}} & =\frac{1}{\left(N_{i}^{S C N}-1\right)} \sum_{j=1}^{N_{i}^{S C N}}\left(V_{j}-\bar{x}_{i}^{v}\right) \\
s_{i}^{p, S C N_{i}} & =\frac{1}{\left(N_{i}^{S C N}-1\right)} \sum_{j=1}^{N_{i}^{S C N}}\left(P_{j}-\bar{x}_{i}^{p}\right)
\end{aligned}
$$

where $s_{i}^{v, S C N_{i}}$ is the standard deviation of speed value for its $S C N_{i}$ and $s_{i}^{p, S C N_{i}}$ is the standard deviation of position value for its $S C N_{i}$.

$$
\begin{aligned}
& z_{i}^{v}=\left(V_{i}-\bar{x}_{i}^{v}\right) / s_{i}^{v, S C N_{i}} \\
& z_{i}^{p}=\left(P_{i}-\bar{x}_{i}^{p}\right) / s_{i}^{p, S C N_{i}}
\end{aligned}
$$

and then $w_{i}$ is;

$$
w_{i}=\left|z_{i}^{v}\right|+\left|z_{i}^{p}\right|
$$

As shown, a node which has shorter distances between the nodes in its $S C N_{i}$, closer speed to the mean speed value of its $S C N_{i}$, and higher $N^{S C N}$ will have more chance to be a $\mathrm{CH}$. 

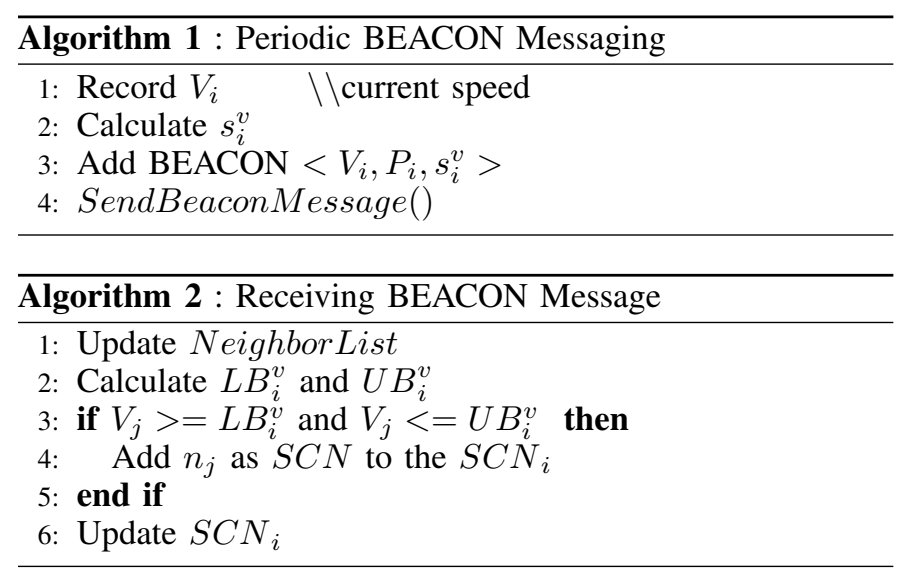

\section{Cluster Head Selection and Cluster Formation}

As defined previously, each vehicle periodically beacons its details containing Node ID, direction, speed, standard deviation of its speed, position (Algorithm 1). On beacon receptions (Algorithm 2), each node updates its neighboring table and $S C N$ list $\left(S C N_{i}\right)$. On re-clustering period, Algorithm 3 is applied at nodes. First, nodes calculates their coherence value to become a $\mathrm{CH}$. Based on this value, a node determines the waiting time $t_{i}^{\text {wait }}$ before broadcasting its $\mathrm{CH}$ declaration message. In case it receives any $\mathrm{CH}$ message from a node in its SCN list before its $t_{i}^{\text {wait }}$ expires, it cancels its $\mathrm{CH}$ declaration message and changes its status as cluster member. Otherwise, on expire of its waiting time $t_{i}^{\text {wait }}$, it broadcast its $\mathrm{CH}$ message to declare itself as $\mathrm{CH}$.

\section{Simulations And Performance Results}

The performance of the proposed approach has been evaluated in a realistic VANET simulation environment consists of OMNeT ++ [12], MiXiM [13], Veins [14], and SUMO [15]. OMNeT++ network simulator and MiXiM framework are used for simulating the network and the communication layers including wireless extensions e.g. radio interference and shadowing. SUMO is used to create traffic simulation scenarios and mobility of the vehicles in detail including the parameters of vehicle maximum speed, acceleration, deceleration and length. Veins couples the two simulators and provides interaction between vehicles and inter-vehicle communication.

TABLE II. SIMULATION PARAMETERS
\begin{tabular}{|l|l|}
\hline Metrics & Value \\
\hline Simulation Area & Highway \\
\hline Road Length & $10 \mathrm{~km}$ \\
\hline Number of Lanes & 4 lanes \\
\hline Number of Vehicles & $150,200,250,300$ \\
\hline Vehicle Speed & $40 \mathrm{~km} / \mathrm{h}-120 \mathrm{~km} / \mathrm{h}$ \\
\hline Transmission Range & $500 \mathrm{~m}$ \\
\hline Beacon Interval & $1 \mathrm{~s}$ \\
\hline Clustering Interval & $30 \mathrm{~s}$ \\
\hline Simulation Time & $1000 \mathrm{~s}$ \\
\hline Confidence Interval & $95 \%$ \\
\hline
\end{tabular}

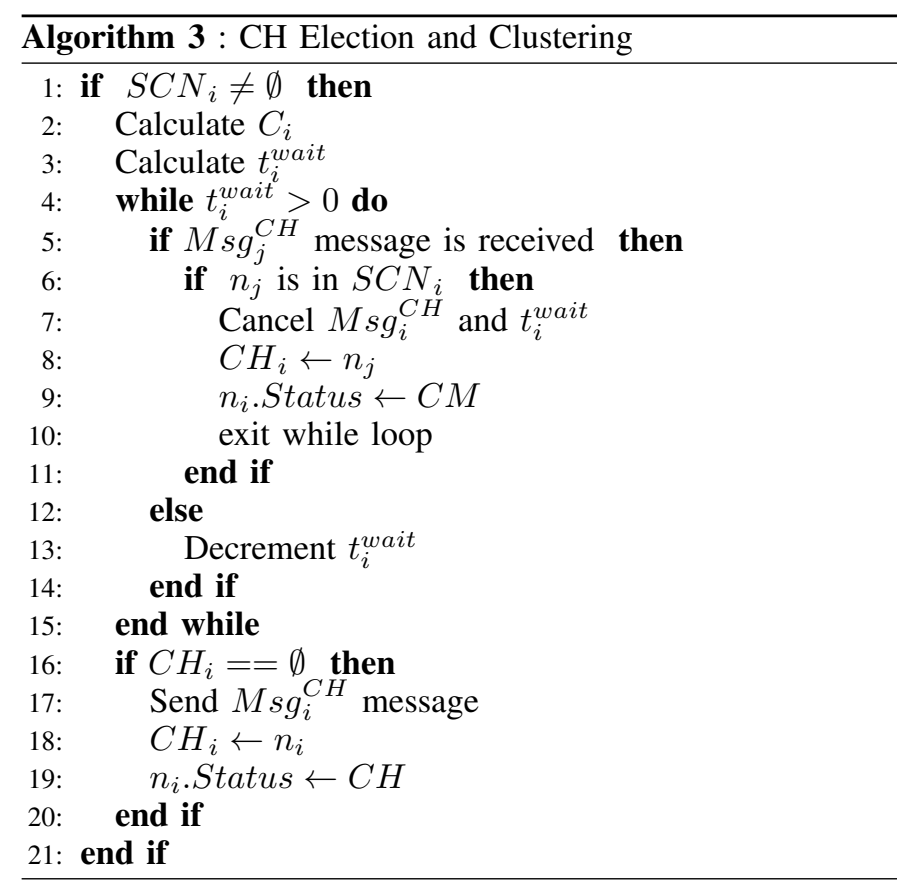

In order to simulate a realistic vehicle pattern in highways, three different speed characteristics are defined for the vehicles as given Table 3. Although the density of vehicles in the network varies in various scenarios, the ratios in Table III are preserved. Vehicles' speeds and inter-vehicles distances are generated from Normal Distribution to be located on a highway with 4-lanes [11] and $10 \mathrm{~km}$ length (Table II). Other common parameters used in the scenarios are given in Table II. At an instant time of the simulations, the number of nodes e.g. 150, is kept constant on $10 \mathrm{~km}$ highway where nodes may leave the system and new ones enter. Performance results are observed in the simulation environment defined above with the standards defined for VANET e.g. IEEE 802.11p.

TABLE III. VEHICLE SPEED CHARACTERISTICS AND THEIR RATIOS
\begin{tabular}{|l|c|c|c|}
\hline & $\begin{array}{c}\text { Min } \\
\text { Speed } \\
(\mathrm{km} / \mathrm{h})\end{array}$ & $\begin{array}{c}\text { Max } \\
\text { Speed } \\
(\mathrm{km} / \mathrm{h})\end{array}$ & $\begin{array}{c}\text { Ratio of the } \\
\text { vehicles } \\
\text { in overall }(\%)\end{array}$ \\
\hline High Speed Vehicles & 90 & 120 & 34 \\
\hline Moderate Speed Vehicles & 60 & 90 & 33 \\
\hline Low Speed Vehicles & 40 & 60 & 33 \\
\hline
\end{tabular}

\section{A. Compared Algorithms and Performance Metrics}

In order to measure the performance of the proposed approach, two well-known algorithms and another competing algorithm are selected for comparison. Lowest-ID and HighestDegree are the two well-known algorithms which are usually compared for benchmarking. Lowest-ID clustering approach uses ID of the nodes to determine the CH. Although it doesn't use essential parameters e.g. speed, it sometimes present sustainable results. Highest-Degree on the other hand uses one important parameter, the density of the nodes, for the election of $\mathrm{CH}$. Since the density is a common parameter in the proposed approach, ReSCUE, the effects of other parameters in the cluster head selection can be seen in the comparison. The third compared algorithm is the Threshold Based (TB) 
clustering approach which is very similar to the proposed approach. As it is discussed in the related work, TB approach doesn't go for $\mathrm{CH}$ election even there are new node arrivals and departures to/from the cluster. $\mathrm{CH}$ election occurs only when there are cluster merges. For the cluster head change rate and cluster head duration, it performs well and is a good competitor for the proposed approach.

Two major performance metrics are measured in the simulations. These are the cluster head change and the cluster head duration which generally show the stability of the clusters. Cluster head change is a metric that shows $\mathrm{CH}$ change frequency of the nodes. In a stable network, $\mathrm{CH}$ change should not occur if the circumstance and conditions don't raise such a need. In a mobile environment such as VANET, $\mathrm{CH}$ change may occur frequently depending on the parameters involved in the $\mathrm{CH}$ election. $\mathrm{CH}$ change causes nodes to choose a new appropriate $\mathrm{CH}$ which overall introduces overhead and change in the topology. Therefore, one of the main aim in stable clustering is reducing the $\mathrm{CH}$ change frequency and therefore $\mathrm{CH}$ change rate. $\mathrm{CH}$ change rate can be calculated as follows where $N^{C H}$ is the total number of nodes which acts as cluster head during the simulation and $n_{j}$ is the node which acts as cluster head:

$$
C H \text { Change Rate }=\frac{1}{N^{C H}} \sum_{j=1}^{N^{C H}} \# \text { of } C H \text { change of } n_{j}
$$

On the other hand, it is essential to prolong lifetime of the clusters as long as possible. When $\mathrm{CH}$ changes occur, nodes register to the new and best appropriate $\mathrm{CH}$ node forming new clusters while changing their states. It means that hierarchy in the topology changes as there are changes in the CHs. To avoid unnecessary and instantaneous changes in the topology, $\mathrm{CHs}$ should be elected considering the consequences of these consecutive events. Hence, cluster head duration becomes an important metric as a measure of stability. It can be calculated as follows:

$$
\text { Av. CH Duration }=\frac{\sum_{j=1}^{N^{C H}} C H \text { duration of } n_{j}}{\sum_{j=1}^{N^{C H}} \# \text { of } C H \text { change of } n_{j}}
$$

\section{B. Performance Results}

Fig.1 shows the average number of cluster head change and Fig. 2 shows the average number of clusters in varying node densities. It is seen in Fig.1 that Lowest-ID and ReSCUE have closer number of $\mathrm{CH}$ changes for the node densities 150 and 200. But, as the density increases, Lowest-ID shows more $\mathrm{CH}$ changes with respect to ReSCUE where ReSCUE performs better than all approaches. In the cluster formation, Lowest-ID doesn't consider the similarities of the nodes. This causes Lowest-ID to include more nodes in the clusters (Fig.5) but less number of clusters (Fig.2). On the other hand, the proposed approach, ReSCUE forms more and smaller size clusters (Fig.5) compared to Lowest-ID which in return causes more $\mathrm{CH}$ change. The number of $\mathrm{CH}$ change is absolutely dependent to the number of formed clusters. Therefore, it is essential to observe the $\mathrm{CH}$ change rate as given in Fig.3.

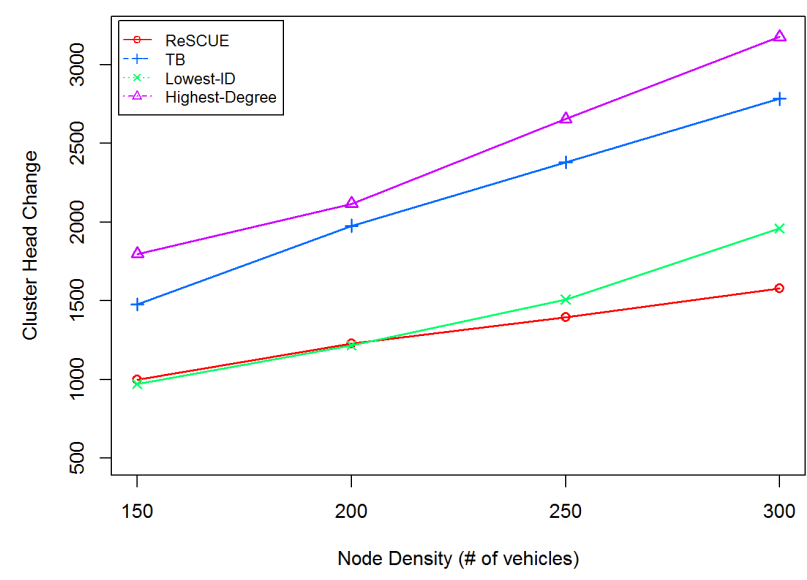

Fig. 1. Number of Cluster Head Change values in various node densities.

In Fig.3, all algorithms show similar patterns but varying values of $\mathrm{CH}$ change rates. Highest-Degree has the highest rate of $\mathrm{CH}$ change. The reason can be explained as follows. When the vehicle groups/clusters (whether or not they have similar characteristic nodes or not) meet with each other, merging will start and the density of the vehicles will increase instantly. Consequently, because the Highest-Degree considers only the density of vehicles for clustering, $\mathrm{CH}$ changes occur frequently. Compared to the Highest-Degree, TB considers more parameters including the density. However, it suffers from the same problem. Because the vehicles in different clusters in TB are not discriminated according to their characteristics when clusters meet, node density becomes an important factor in $\mathrm{CH}$ election and clustering. Therefore, $\mathrm{CH}$ changes occur frequently depending on the density and meeting frequency of the clusters. On the other hand, the effect of node density is not seen in Lowest-ID, because it doesn't consider the density in $\mathrm{CH}$ election. Interestingly, $\mathrm{CH}$ change is very low in LowestID. The reason is that a portion of the CHs with the lowest-ID is again becoming the node with the lowest-ID when clusters meet with each other. Therefore, $\mathrm{CH}$ change in Lowest-ID is low compared to Highest-Degree and TB. ReSCUE has the minimum rate of $\mathrm{CH}$ change among all approaches. Similar to the Highest-Degree and TB, ReSCUE considers the density of the nodes in clustering. However, the density doesn't cause changes of CHs if the conditions are not met in ReSCUE. Because the ReSCUE discriminates and segregates the clusters based on their node characteristics, the meet of clusters doesn't affect the clusters if there is no common characteristics among the clusters.

Similar results are observed on the duration of $\mathrm{CH}$ as shown in Fig.4. Highest-Degree has the smallest $\mathrm{CH}$ duration for all varying density values. The density of the vehicles increases abruptly when the clusters meet with each other and decreases abruptly when the faster nodes leave the cluster, splitting the cluster again. This behavior affects the $\mathrm{CH}$ duration in Highest-Degree. Similar effects are seen in Lowest-ID. Because Lowest-ID doesn't consider any parameter other than ID, cluster head changes occur only if there are nodes with ID smaller than the ID of CHs when the clusters meet. In this case, similar to the Highest-Degree, CHs instantaneously change when clusters merge and split, which eventually affects the duration of $\mathrm{CH}$. TB presents longer duration of $\mathrm{CH}$ compared to Highest-Degree and Lowest-ID. ReSCUE performs 


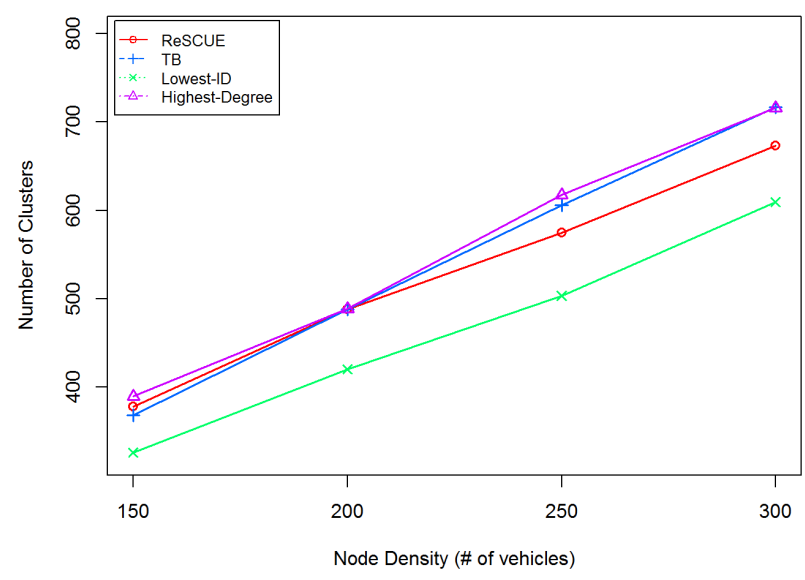

Fig. 2. Number of Clusters in various node densities.

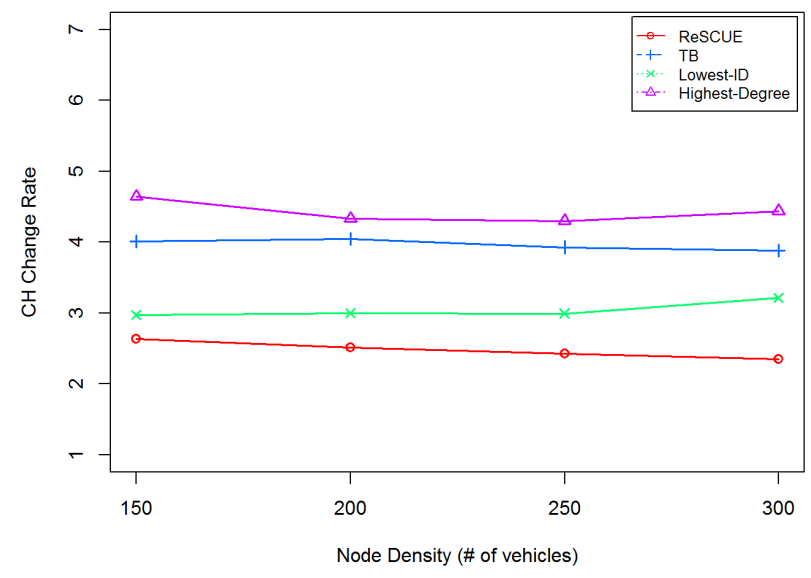

Fig. 3. Cluster Head Change rates in various node densities.

better than all approaches with longer and stable duration of CHs. The main reasons as aimed in this approach are first, discrimination and segregation of the clusters depending on their characteristics to avoid unnecessary cluster merges, and secondly, forming more balanced clusters considering the spatio-temporal changes in node characteristics and the coherency of nodes.

The average number of nodes per cluster and its $95 \%$ confidence interval (for the scenario with 250 nodes) are shown in Fig.5. The number of nodes per cluster varies too much for the Lowest-ID and Highest-Degree for the same reasons given previously. When clusters meet, Lowest-ID and Highest-Degree approaches form new clusters by merging the clusters. On the other hand, ReSCUE and TB forms more stable clusters because they consider the similarities of nodes in cluster formation. Average number of cluster member in TB is very low, because it is observed that many nodes are not included in cluster while moving as standalone nodes as defined in [9]. ReSCUE forms more stable clusters in terms of the number of member nodes and the variation of the number of member nodes.

The results highlight the fact that clusters have to be merged only if the conditions are suitable to merge. In a frequently changing topology with various vehicle types and with different characteristics, clusters have to be formed considering these characteristics. ReSCUE satisfies this requirement in

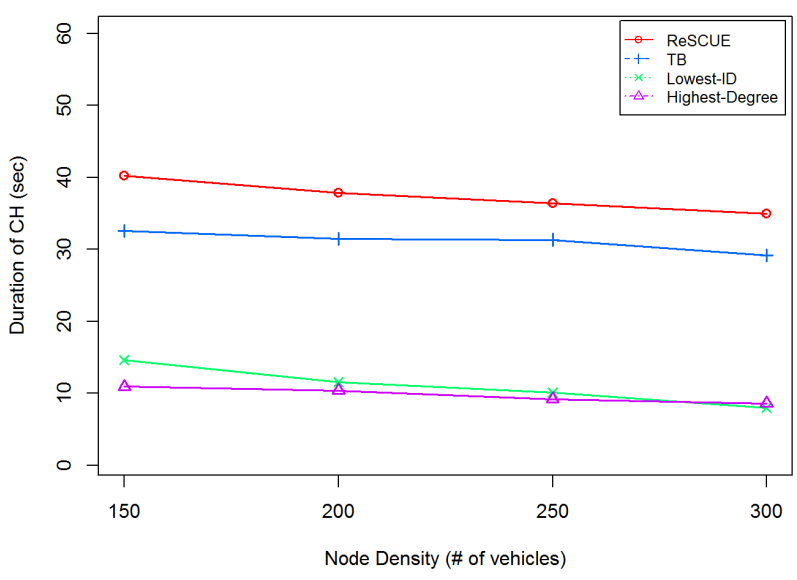

Fig. 4. Average Cluster Head Duration.

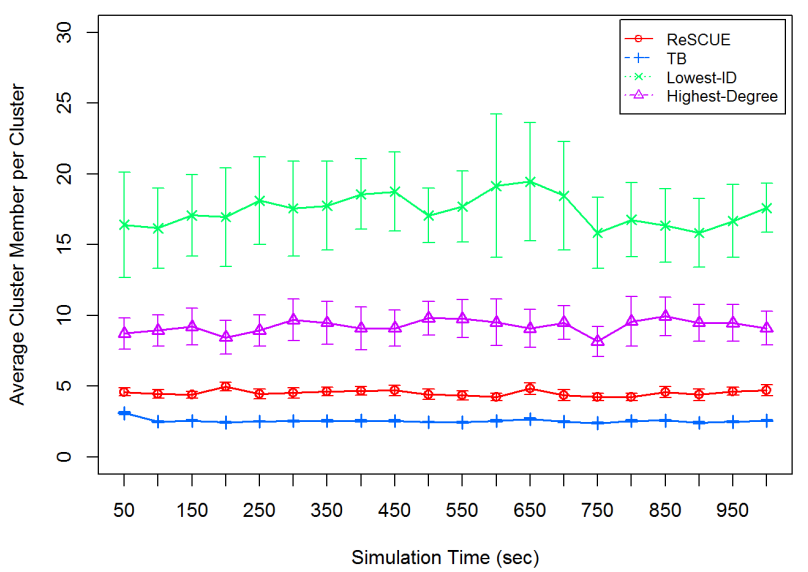

Fig. 5. Average Cluster Member per Cluster for the scenario with 250 nodes.

addition to the approach that discriminates clusters to prevent unnecessary merges.

\section{CONCLUSION}

Clustering algorithms in VANET consider various parameters in cluster formation and cluster head election. However, there is general approach to merge clusters when they meet with each other. In this paper, it is discussed and demonstrated that merging clusters without considering the circumstances and common characteristics of the clusters degrades the stability of the clusters. The proposed approach, ReSCUE prevents clusters to merge by considering the characteristics of the clusters. ResCUE significantly reduces cluster head change and enhances the cluster head duration while forming more stable clusters. The main contributions of the ReSCUE are in twofold: first, it discriminates the vehicles and clusters to avoid unnecessary merges and secondly, it considers the local information and current characteristics of the vehicles in making decisions without biasing by using general characteristics and the preknown information of the vehicles in the network. As far as we know, ReSCUE is the first clustering approach in the literature which aims to avoid unnecessary cluster merging. In our test, we have also observed that ReSCUE has low communication overhead with respect to compared protocols. Detailed analysis and the performance evaluation of the ReSCUE in terms of communication are left as future works. 


\section{REFERENCES}

[1] S. Al-Sultan, M. M. Al-Doori, A. H. Al-Bayatti, and H. Zedan, "A comprehensive survey on vehicular ad hoc network," Journal of Network and Computer Applications, vol. 37, no. 0, pp. 380 - 392, 2014.

[2] A. Parekh, "Selecting routers in ad-hoc wireless networks," ITS, 1994.

[3] A. Ephremides, J. Wieselthier, and D. Baker, "A design concept for reliable mobile radio networks with frequency hopping signaling," Proceedings of the IEEE, vol. 75, no. 1, pp. 56-73, Jan 1987.

[4] P. Basu, N. Khan, and T. Little, "A mobility based metric for clustering in mobile ad hoc networks," in Distributed Computing Systems Workshop, 2001 International Conference on, Apr 2001, pp. 413-418.

[5] E. Souza, I. Nikolaidis, and P. Gburzynski, "A new aggregate local mobility (ALM) clustering algorithm for vanets," in Communications (ICC), 2010 IEEE International Conference on, May 2010, pp. 1-5.

[6] Z. Zhang, A. Boukerche, and R. Pazzi, "A novel multi-hop clustering scheme for vehicular ad-hoc networks," in Pr. of the ACM Int. Symp. on Mobility Management and Wireless Access, MobiWac'11, 2011.

[7] C.-C. Chiang and M. Gerla, "Routing and multicast in multihop, mobile wireless networks," in Universal Personal Communications Record. Conference Record., IEEE Int.Conf. on, 1997.

[8] K. Xu and M. Gerla, "A heterogeneous routing protocol based on a new stable clustering scheme," in MILCOM 2002. Proceedings, vol. 2, Oct 2002, pp. 838-843 vol.2.

[9] Z. Rawashdeh and S. M. Mahmud, "A novel algorithm to form stable clusters in vehicular ad hoc networks on highways," EURASIP Journal on Wireless Communications and Networking, vol. 2012, no. 1, 2012.

[10] M. Rudack, M. Meincke, K. Jobmann, and M. Lott, "On traffic dynamical aspects of inter vehicle communications (ivc)," in IEEE 58th VTC 2003-Fall, 2003.

[11] A. Mehar, S. Chandra, and S. Velmurugan, "Speed and acceleration characteristics of different types of vehicles on multi-lane highways," Trasporti europei (Online), no. 55, 2013.

[12] A. Varga, "Using the omnet++ discrete event simulation system in education," IEEE Trans. on Education, vol. 42, no. 4, p. 11, 1999.

[13] A. Köpke et al., "Simulating wireless and mobile networks in omnet++ the mixim vision," in Pr. of Int.Conf. on Simu. Tools and Techniques for Comm., Networks and Systems \& Workshops, Simutools '08, 2008.

[14] C. Sommer, R. German, and F. Dressler, "Bidirectionally coupled network and road traffic simulation for improved ivc analysis," Mobile Computing, IEEE Transactions on, vol. 10, no. 1, pp. 3-15, Jan 2011.

[15] D. Krajzewicz et al., "Recent development and applications of SUMO - Simulation of Urban MObility," Int. Journal On Advances in Systems and Measurements, vol. 5, no. 3\&4, pp. 128-138, Dec. 2012. 